\title{
Numerical Modeling of the Photothermal Processing for Bubble Forming around Nanowire in a Liquid
}

\author{
Anis Chaari, Laurence Giraud-Moreau, Thomas Grosges, and Dominique Barchiesi \\ Group for Automatic Mesh Generation and Advanced Methods, Gamma3 (UTT-INRIA), University of Technology of Troyes, \\ 12 rue Marie Curie, CS 42060, 10004 Troyes Cedex, France
}

Correspondence should be addressed to Thomas Grosges; thomas.grosges@utt.fr

Received 2 January 2014; Accepted 4 March 2014; Published 24 March 2014

Academic Editors: D. Baleanu and H. Jafari

Copyright (C) 2014 Anis Chaari et al. This is an open access article distributed under the Creative Commons Attribution License, which permits unrestricted use, distribution, and reproduction in any medium, provided the original work is properly cited.

\begin{abstract}
An accurate computation of the temperature is an important factor in determining the shape of a bubble around a nanowire immersed in a liquid. The study of the physical phenomenon consists in solving a photothermic coupled problem between light and nanowire. The numerical multiphysic model is used to study the variations of the temperature and the shape of the created bubble by illumination of the nanowire. The optimization process, including an adaptive remeshing scheme, is used to solve the problem through a finite element method. The study of the shape evolution of the bubble is made taking into account the physical and geometrical parameters of the nanowire. The relation between the sizes and shapes of the bubble and nanowire is deduced.
\end{abstract}

\section{Introduction}

In the last years, many researchers are interested in the use of nanomaterials. In the chemical industry and in the manufactures of nanotubes and nanowires, the usually used materials are $\mathrm{TiO}_{2}$ and $\mathrm{ZnO}$ [1-3]. Such a use of these nanomaterials (natural or artificial) increases and these are dispersed in air or in water [4]. Their impact on the environment and health must be evaluated (e.g., toxicity analysis) [5]. Therefore, the detection of the presence of such nanomaterials in the environment becomes crucial. Two modes of detections of such a nanowire/nanotube can be achieved. The first one consists in a direct detection of the nanowire by optical microscopy through the measurement of the scattering of light emitted by the nanomaterial. Due to a weak signal/noise ratio, such a detection mode can be difficult. The second mode is an indirect method and consists in studying the bubble created by the photothermal response of the nanowire immersed in a liquid and illuminated by an electromagnetic wave. In such an approach, the nanowire absorbs the electromagnetic radiation (energy) for a range of wavelengths and heats and, for temperature exceeding the threshold of vaporization of the liquid, induces the creation of a nanobubble $[6,7]$. The created bubble grows before being detected. The analysis of the shape and size of the bubble should permit studying the morphology of the nanowire. The studied problem consists in solving a photothermic coupled system (light, nanowire, and heat) taking into account the physical parameters of the system (i.e., permittivity of materials, material conductivity, laser wavelength, and laser power).

In that context, a numerical multiphysic model, allowing studying the behavior of the nanowires illuminated by an incident laser field, is presented. The formation of the bubble, associated with a nanowire of $\mathrm{TiO}_{2}$ immersed in water and illuminated by a laser pulse, is studied. An optimization process, including adaptive remeshing scheme, is used to detect the variations of the temperature, the bubble shape evolution, ensuring the convergence of the solution to the physical solution $[8,9]$. The paper is organized as follows. Section 2 describes the equations of the model and the numerical resolution method. The adaptive remeshing process and the optimization steps are presented in Section 3. In Section 4, the results of numerical simulations are presented before concluding.

\section{Model and Numerical Methods}

The section is devoted to presenting the equation systems modeling the photothermic process and the numerical method used to solve the system. 
2.1. Electromagnetic Problem. In electromagnetic system, the partial differential equations are derived from Maxwell's equations. The problem can be reduced to Helmholtz equation for the harmonic electric $\mathbf{E}$ and magnetic $\mathbf{H}$ fields (i.e., in the form $\exp (j \omega t)$, where $\omega$ is the angular frequency of the harmonic wave) [10]. In the $2 \mathrm{D}$ case of an infinity elliptical cylinder along the $z$-axis, the unknown field is the magnetic component and, for a polarized illumination in the transverse magnetic mode TM, the magnetic field can be written as $\mathbf{H}(x, y)=\left(0,0, H_{z}(x, y)\right)$. Therefore, the electromagnetic problem is reduced to a scalar problem and the computation of $\mathbf{H}(x, y)$, in a domain $\Omega$, allows deducing the electric field $\mathbf{E}(x, y)$ by using the Maxwell-Ampere equation [10]:

$$
\mathbf{E}(x, y)=\frac{-j}{\omega \epsilon_{r} \epsilon_{0}}[\nabla \times \mathbf{H}(x, y)], \quad \forall(x, y) \in \Omega,
$$

where $(\nabla \times \cdot)$ is the rotational operator, $\omega$ the angular frequency, $\epsilon_{0}$ the permittivity of vacuum, and $\epsilon_{r}$ the relative complex permittivity of the considered materials which are functions of the spatial coordinates $(x, y)$. The $H_{z}$ component of the magnetic field satisfies the scalar equation

$$
\left[\nabla \cdot\left(\frac{1}{\epsilon_{r}} \nabla\right)+k_{0}^{2}\right] H_{z}(x, y)=0, \quad \forall(x, y) \in \Omega,
$$

where $k_{0}=\omega / c$ is the wave number of the monochromatic incoming wave and $c$ the speed of light in vacuum. To compute the solution $H_{z}(x, y)$ of the electromagnetic problem, a set of conditions on the boundary $\Gamma$ of the computational domain $\Omega$ must be imposed. The natural boundary condition at the interface between materials is the continuity of the normal component of the electromagnetic excitation:

$$
\begin{array}{r}
\frac{1}{\epsilon_{r}} \frac{\partial H_{z}(x, y)}{\partial n}=-j k_{0}\left[H_{z}(x, y)-\left(n_{y}-1\right) H_{i}(x, y)\right], \\
\forall(x, y) \in \Gamma,
\end{array}
$$

where $\partial / \partial n$ is the normal derivative operator, $n_{y}$ is the normal vector component along the $y$-axis, and $H_{i}=H_{0} \exp \left(j k_{0} y\right)$ is the incident illumination field along the $y$-axis with $H_{0}=$ $1 /\left(c \mu_{0}\right)$ and $\mu_{0}$ being the permeability of vacuum. Such a boundary condition is used in problems of wave propagation [11-13].

2.2. Thermic Problem. Under illumination by an electromagnetic wave, the nanowire absorbs energy. That energy produces a heat source given by

$$
Q(x, y)=\frac{\omega}{2} \epsilon_{0} \operatorname{Im}\left(\epsilon_{r}\right)|\mathbf{E}(x, y)|^{2}, \quad \forall(x, y) \in \Omega .
$$

The resolution of the thermal problem requires solving the heat equation which is a partial differential parabolic equation describing the evolution of the temperature $T$ with a heat source $Q$. That equation is written as follows:

$$
[\nabla \cdot(k(x, y) \nabla)] T(x, y)=Q(x, y), \quad \forall(x, y) \in \Omega,
$$

with a Dirichlet boundary condition $T=T_{0}$ and $k(x, y)$ is the thermal conductivity of the materials. The variation of the temperature depends on both imaginary part of the permittivity $\epsilon_{r}(x, y)$ and the intensity of the electric field $|\mathbf{E}(x, y)|^{2}$.

The resolution of the coupled electromagnetic and heat problems allows extracting the spatial distribution of the temperature in the computational domain. From the map of temperature and for a fixed threshold of vaporization $\alpha$, the identification of the shape and size of the bubble around the nanowire can be achieved. Such information on shape and size of the bubble would be used to construct a relation between the geometric characteristics of the bubble and the nanowire.

2.3. The Finite Element Method. The objective is to solve (2) and (5) for the coupled system in a domain whose geometry can be complex. The Finite Element Method (FEM) was applied since the 1940s in mechanics, thermodynamics, electromagnetics, and electrical engineering [14, 15]. The method is used to solve partial differential equation systems with boundary conditions in open or close domains. The resolution of problem necessitates a discrete domain, generally named mesh of the domain [13]. The solutions of the problem are computed on the nodes of the mesh. In order to both control the error on the solution and to decrease the number of nodes, an improved method, including an iterative remeshing process, is developed and used. Such an improved FEM allows describing the complex structures with arbitrary shapes. Moreover, the stability of the FEM is also improved by using a weak formulation (or variational formulation) of (2) and (5). Therefore, the electromagnetic and thermic fields satisfy

$$
\begin{gathered}
\int_{\Omega}\left[\nabla \cdot\left(\frac{1}{\epsilon_{r}} \nabla H_{z}(x, y)\right)+\frac{\omega^{2}}{c^{2}} H_{z}(x, y)\right] \cdot v d \Omega=0, \\
\int_{\Omega}[\nabla \cdot(k(x, y) \nabla) T(x, y)-Q(x, y)] \cdot \widetilde{\nu} d \Omega=0,
\end{gathered}
$$

where $\nu$ and $\widetilde{\nu}$ are test functions defined on $L^{2}(\Omega)$ (the linear space of the scalar functions $\nu$ and $\widetilde{\nu}$, being 2-integrable on $\Omega$ ). The basis of polynomial functions provides an approximation of the solutions $H_{z}$ and $T$ in each node [16]. The field $H_{z}$ (resp., $T$ ) is a linear combination of such basic polynomial functions $v$ (resp., $\widetilde{v}$ ) and the problem consists in solving a linear system $[15,17]$. The solution verifies exactly the partial differential equations on each node for the given boundary conditions. Ritz's formulation of the variational problem is used to satisfy the continuity of the tangential components of the electromagnetic field [13].

\section{Optimization Process and Adaptive Remeshing}

Partial differential equations (electromagnetic and thermic) are formulated and solved on the mesh of the computational domain through the FEM. But the accuracy of the computed solution depends on the quality of the mesh $[9,18,19]$. 
A remeshing process and adaptive loops have been developed in order to improve the quality of the solutions by adapting the size of the mesh elements to the physical solution $[9,20]$. The mesh adaption is required to converge to a stable solution, in particular where strong variation of the electromagnetic or temperature fields occurred. For each step of the adaption process, the approximate solution of the Helmholtz equation, the electric field E, the heat source $Q$, and the temperature $T$ are computed [20]. The interpolation error, based on an estimation of the discrete Hessian of the solution, is used to limit the maximum deviation between the exact solution and the solution associated with the mesh $[21,22]$. The a posteriori error estimator, based on the interpolation error, allows defining a physical size map $C_{p}(\Omega)$ such as

$$
C_{p}(\Omega)=\left\{h_{p}(x, y)\right\}, \quad \forall(x, y) \in \Omega,
$$

where $h_{p}(x, y)$ is the physical size defined at each node and is proportional to the inverse of the deviation of the Hessian. For a given maximum tolerance on the physical error $\gamma$, the size $h_{p}(x, y)$ is given by

$$
h_{\min } \leq h_{p}(x, y)=\frac{\gamma}{\eta(x, y)} \leq h_{\max }
$$

where $h_{\min }$ and $h_{\max }$ are the minimum and maximum sizes of the elements and $\eta(x, y)$ is an estimation of the maximum deviation obtained from the Hessian of the solution. The physical size map $C_{p}(\Omega)$ is used to govern the adaptive remeshing of the domain with the BL2D-V2 software (adaptive remeshing generating isotropic or anisotropic meshes) [23]. The domain is then entirely remeshed and a new mesh $M_{p}(\Omega)$ is obtained. The resolution of the multiphysics problem is based on the computation of two physical size maps: the first one $C_{Q}(\Omega)$ related to the heat source $Q$ and the second one $C_{T}(\Omega)$ related to the temperature $T$. The adaptive computational scheme consists in iterative and adaptive loops:

$A_{1}$ initial mesh $M_{i=0}(\Omega)$ generated with triangular elements of the computational domain $\Omega$,

$A_{2}$ computation of the field $\left(H_{z}\right)_{i}$ (solution of (2)) on $M_{i}(\Omega)$,

$A_{3}$ computation of the solutions $\mathbf{E}_{i}$ and $Q_{i}$ on $M_{i}(\Omega)$,

$A_{4}$ physical error estimation: computation of the interpolation error of the physical solution $Q_{i}$; definition of a physical size map $C_{Q_{i}}(\Omega)$ connected to the field $Q_{i}$ enabling relating the error to a given threshold $\delta$,

$A_{5}$ remeshing of the domain conforming to the size map $C_{Q_{i}}(\Omega)$,

$A_{6}$ if the threshold $\delta$ is not satisfied loop to step $A_{2}$, with $i=i+1$, in order to obtain a new mesh $M_{i}(\Omega)$, else $M_{Q}(\Omega)=M_{i}(\Omega)$, and $M_{i=0}(\Omega)=M_{Q}(\Omega)$,

$B_{1}$ computation of the solutions $T_{i}$ on $M_{i}(\Omega)$,

$B_{2}$ physical error estimation: computation of the interpolation error of the physical solution $T_{i}$; definition of a physical size map $C_{T_{i}}(\Omega)$ connected to the field $T_{i}$ enabling relating the error to a given threshold $\delta$,

$B_{3}$ remeshing of the domain conforming to the size map $C_{T_{i}}(\Omega)$,

$B_{4}$ if the threshold $\delta$ is not satisfied loop to step $B_{1}$, with $i=i+1$, in order to compute the temperature $T_{i}$ on the new adapted mesh $M_{i}(\Omega)$,

else $M_{T}(\Omega)=M_{i}(\Omega)$,

$C_{1}$ detection of the new domain (water vapor) on $M_{T}$ for a fixed threshold of vaporization in order to produce a mesh $M_{V}(\Omega)$ and $M_{i=0}(\Omega)=M_{V}(\Omega)$,

$C_{2}$ computation of the physical solutions $T_{i}$ on $M_{i}(\Omega)$,

$C_{3}$ physical error estimate: computation of the interpolation error of the physical solution $T_{i}$; definition of a physical size map $C_{V_{i}}(\Omega)$ connected to the field $T_{i}$ enabling relating the error to a given threshold $\delta$,

$C_{4}$ remeshing of the domain conforming to the size map $C_{V_{i}}(\Omega)$,

$C_{5}$ if the threshold $\delta$ is not satisfied loop to step $C_{2}$, with $i=i+1$, in order to compute the temperature $T_{i}$ on the last adapted mesh $M_{i}(\Omega)$,

else $M_{F}(\Omega)=M_{i}(\Omega)$.

\section{Numerical Results and Discussion}

Here, we consider a $\mathrm{TiO}_{2}$ elliptical nanowire of semiaxes ( $a=45 \mathrm{~nm}$ and $b=10 \mathrm{~nm}$ ), with thermal conductivity $k\left(\mathrm{TiO}_{2}\right)=11.7 \mathrm{Wm}^{-1} \mathrm{~K}^{-1}$ immersed in water $\left(\epsilon_{r}\right.$ (water $)=$ 1.79 and $k$ (water $\left.)=0.6 \mathrm{Wm}^{-1} \mathrm{~K}^{-1}\right)$ at temperature $T_{0}=$ $25^{\circ} \mathrm{C}(298.15 \mathrm{~K})$. The nanowire is illuminated by a TM polarized laser pulse at wavelength $\lambda=1050 \mathrm{~nm}$ of complex permittivity $\epsilon_{r}\left(\mathrm{TiO}_{2}\right)_{1050}=5.4600+j 0.00148$ with a power density per area units $P_{S}=1.75 \times 10^{12} \mathrm{~W} / \mathrm{m}^{2}[24,25]$. The materials of the system are considered isotropic and homogeneous.

The results of the adaptive process on mesh and on the temperature maps are illustrated in Figure 1. Figures 1(a) and 1(b) show the initial mesh $M_{0}$ and the associated temperature. The adaptive process on the temperature field $T$ (with $\gamma=0.0001, h_{\max }=40 \mathrm{~nm}, h_{\min }=0.03 \mathrm{~nm}$, and $\delta=0.1$ ) produces the mesh $M_{T}$ and the temperature map. The mesh is adapted on the outline of the nanowire that presents strong variations of the temperature. For a water vaporization threshold $\alpha=100^{\circ} \mathrm{C}(373.15 \mathrm{~K})$, the detection of the new material (water vapor) is obtained from the temperature map computed on the mesh $M_{T}$. Figure 1(c) presents the areas of the three materials: $\mathrm{TiO}_{2}$ (red), vapor (green), and water (blue). The computation of the temperature on the domain that contains the water vapor requires including the physical parameters of the vapor (permittivity $\epsilon_{r}$ (vap) $=1.79$ and thermic conductivity $k($ vap $\left.)=0.05 \mathrm{Wm}^{-1} \mathrm{~K}^{-1}\right)$. The spatial distribution of the temperature field $T$ on the mesh $M_{V_{0}}$ after detection of 


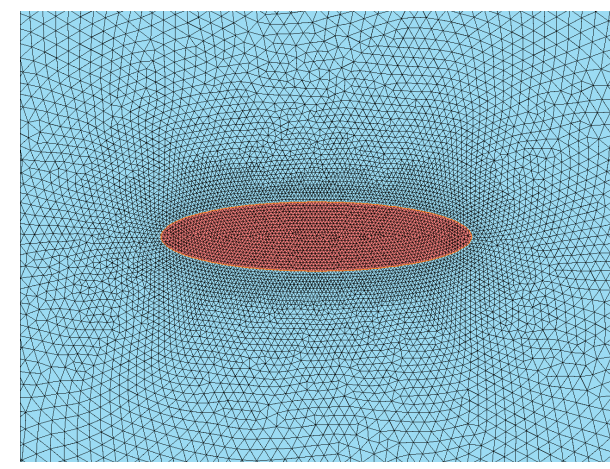

(a)

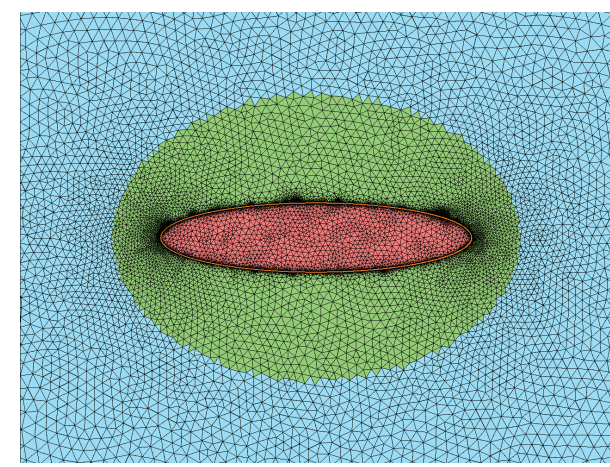

(c)

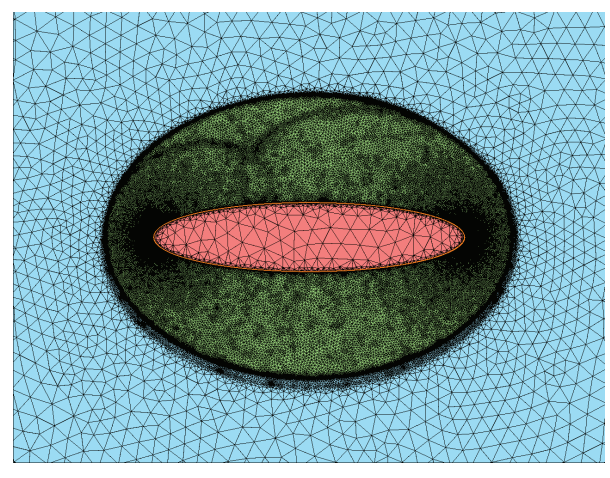

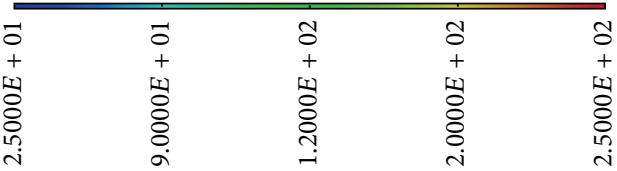

(e)

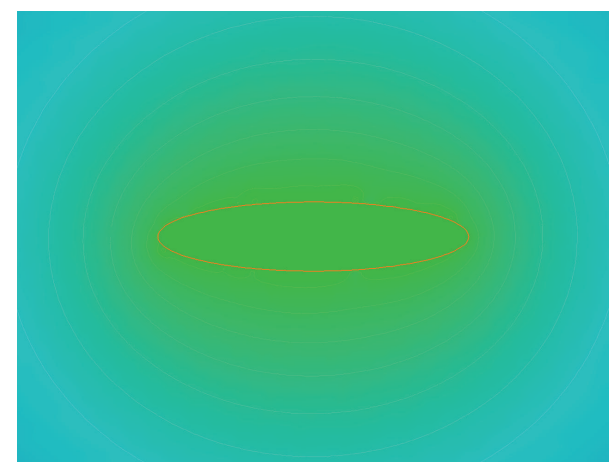

(b)

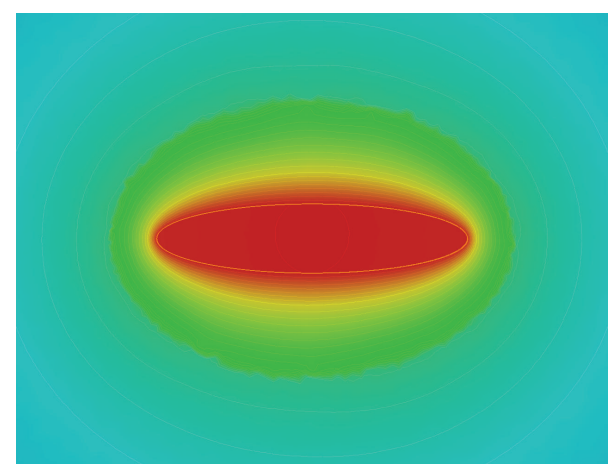

(d)
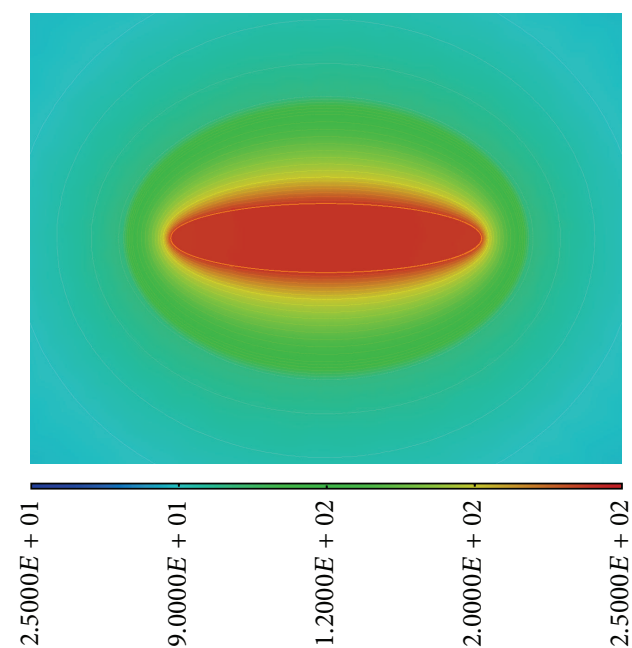

(f)

FIGURE 1: Initial mesh $M_{0}$ (a) and adaptive meshes $M_{V_{0}}(\mathrm{c})$ and $M_{F}(\mathrm{e})$ and the associated temperature maps on $M_{0}(\mathrm{~b}), M_{V_{0}}$ (d), and $M_{F}(\mathrm{f})$ for nanowire illuminated at $\lambda=1050 \mathrm{~nm}$.

the bubble produced around the nanowire is shown in Figure $1(\mathrm{~d})$. The final mesh $M_{F}$ is obtained, after eight iterations, by applying the adaptive process on the field $T$ (with $\delta=$ 0.02 ) taking into account the bubble. That mesh is adapted in the bubble especially on its outline where variations in the temperature occur and relaxed inside the nanowire where the temperature is almost constant (Figure 1(e)). The remeshing process takes into account the shape and size of the bubble. Figure 1(f) shows the temperature map $T$ on the mesh $M_{F}$ after convergence to a stable solution. The level curves are smooth where a strong variation of the temperature is shown (in the vicinity of the boundary of the bubble and the nanowire). The map also shows an increase of the temperature in the nanowire due to the creation of the bubble. Such an increase is due to the diffusion of the temperature, produced by the nanowire after detection of the bubble (i.e., the water vapor has a smaller thermal conductivity than water).

In order to study the evolution of the shape and size of the bubble, we also consider the nanowire illuminated 


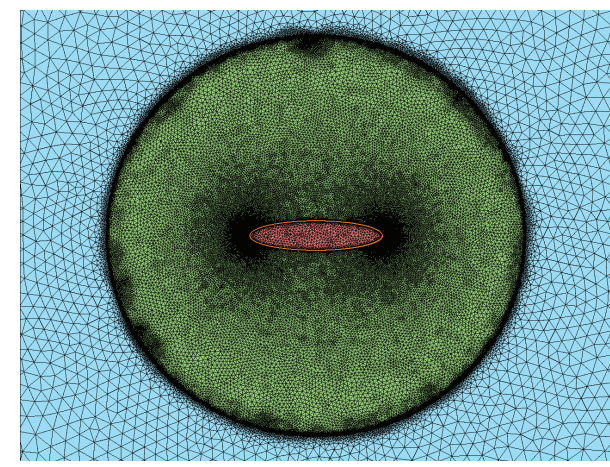

(a)

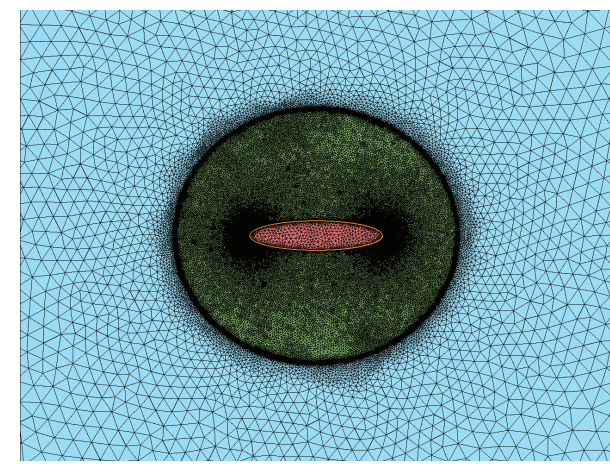

(c)

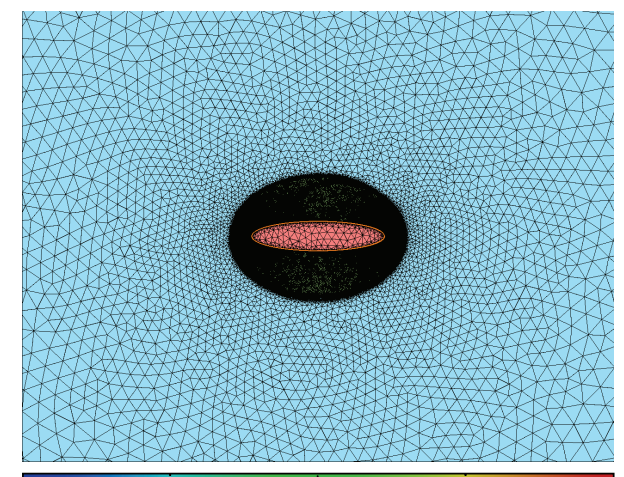

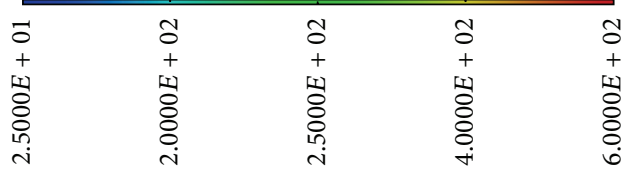

(e)

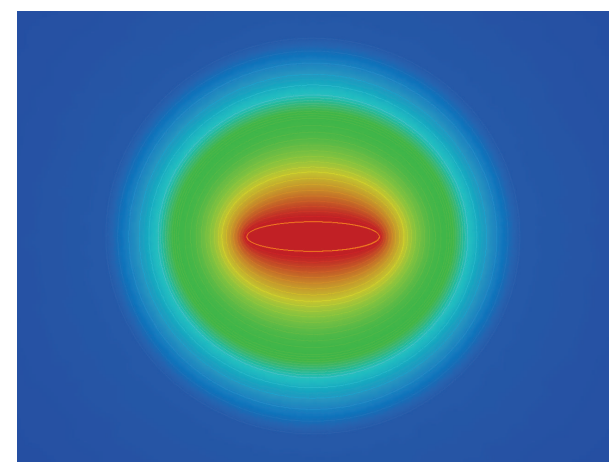

(b)

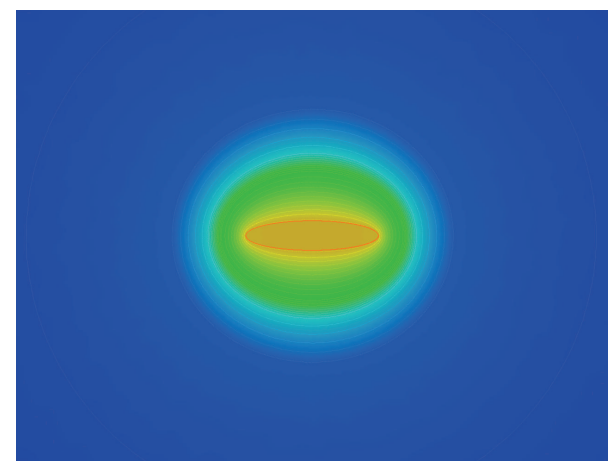

(d)

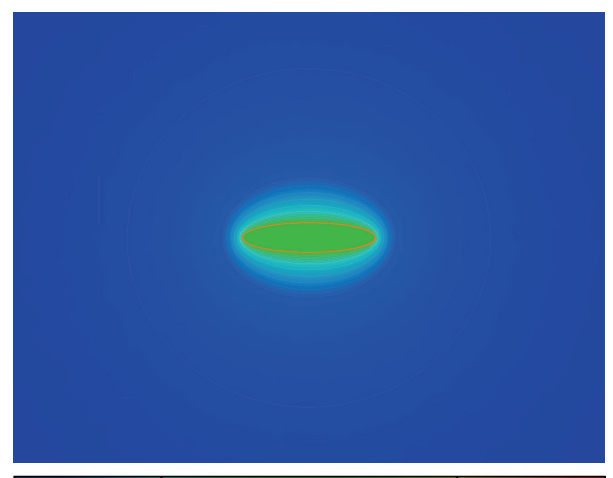

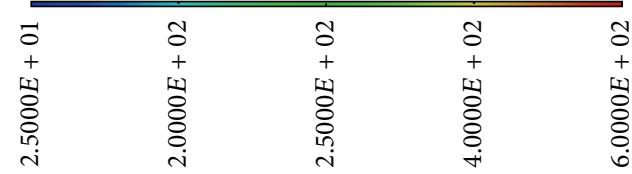

(f)

Figure 2: Adapted meshes $M_{F}\left((\mathrm{a})\right.$, (c), and (e)) and temperature map $T$ on $M_{F}((\mathrm{~b})$, (d), and (f)) for wavelengths $\lambda=950,1000$, and 1050 nm, respectively.

at three wavelengths $\lambda=950 \mathrm{~nm}, \lambda=1000 \mathrm{~nm}$, and $\lambda=1050 \mathrm{~nm}$ with physical parameters $\left(\epsilon\left(\mathrm{TiO}_{2}\right)_{950}=\right.$ $5.500+j 0.00164, \epsilon_{r}\left(\mathrm{TiO}_{2}\right)_{1000}=5.475+j 0.00154$, and $\left.\epsilon_{r}\left(\mathrm{TiO}_{2}\right)_{1050}=5.460+j 0.00148\right)$. Figures 2(a), 2(b), 2(c), 2(d), 2(e), and 2(f) present the mesh $M_{F}$ after bubble detection and distribution of the temperature $T$ on the meshes for the three different wavelengths $\lambda=950 \mathrm{~nm}, \lambda=1000 \mathrm{~nm}$, and $\lambda=1050 \mathrm{~nm}$, respectively. These show the evolution of the meshes (shape and size of the bubble) and the temperature as function of the wavelength. For $\lambda$ increasing, the imaginary part of the complex permittivity of the $\mathrm{TiO}_{2}$ decreases, leading to a decrease of the energy absorbed by the nanowire. Therefore, the temperature also decreases and the shape and size of bubble are changing. The created bubble follows the shape of the nanowire (elliptical) at the beginning and becomes circular with the increase of the temperature. Figure 3 shows the evolution of the mean temperature $T$ in the nanowire as function of the aspect ratio $R_{n}=a / b$ for three wavelengths $\lambda=950,1000$, and $1050 \mathrm{~nm}$ on the mesh $M_{F}$. 


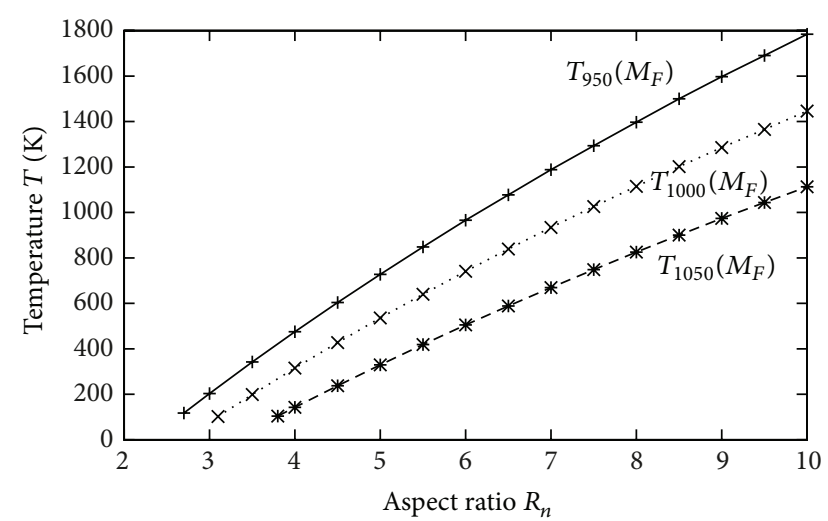

FIGURE 3: Evolution of the mean temperature as function of the nanowire aspect ratio $R_{n}$ after formation of the bubble for the three wavelengths on $M_{F}$.

TABLE 1: Fit parameters of the $f$ function.

\begin{tabular}{lccccccc}
\hline$\lambda$ & \multirow{2}{*}{ NDP } & NDF & FNI & \multicolumn{5}{c}{ Set of parameters } & \multirow{2}{*}{$\sigma^{2}$} \\
& & & & $a_{0}$ & $a_{1}$ & $a_{2}$ & \\
\hline 950 & 42 & 39 & 32 & 1.0036 & 0.2418 & 2.2334 & $1.8111 e-5$ \\
1000 & 38 & 35 & 15 & 1.0082 & 0.3659 & 2.6394 & $5.5850 e-5$ \\
1050 & 31 & 28 & 23 & 1.0124 & 0.6529 & 3.2416 & $1.1106 e-4$ \\
\hline
\end{tabular}

Figure 4(a) shows the evolution of the aspect ratio of the bubble $R_{b}=A / B$ ( $A$ and $B$ being the semiaxes of the bubble) as function of the aspect ratio of the nanowire $R_{n}$ for the three wavelengths. From the computed data, a function $f$, satisfying $R_{b}=f\left(R_{n}\right)$, can be obtained through a nonlinear least-squares fit method (LLS) by the MarquardtLevenberg algorithm [26-29]. The method is used to find a set of the best parameters fitting the data. It is based on the sum of the squared differences or residuals (SSR) between the input data and the function evaluated at the data. The applied algorithm consists in minimizing the residual variance $\sigma^{2}=$ SSR/NDF with NDF being the number of degrees of freedom (number of the data points (NDP) minus number of the estimated parameters) after a finite number of iterations (FNI). Therefore, the function can be written as follows:

$$
f(x)=a_{0}+\frac{a_{1}}{\left(x-a_{2}\right)^{2}},
$$

with $a_{0}, a_{1}$, and $a_{2}$ being set of parameters varying as function of the wavelength. The parameter $a_{0}$ concerns the asymptote value which is related to the maximum ratio for a circular bubble, $a_{1}$ is the inverse of the decay rate of the function $f$ which is related to the speed tending to the circular shape, and $a_{2}$ is the initial ratio from which the bubble begins to form. Table 1 shows the fit parameters for each wavelength. The $f$ function is continuous and strictly decreasing for $R_{n}$ in the interval $] a_{2},+\infty$ [; therefore the inverse function $f^{-1}$ exists. The measurement of the aspect ratio of the bubble $R_{b}$ allows predicting the aspect ratio of the nanowire $R_{n}$ through the relation $R_{n}=f^{-1}\left(R_{b}\right)$. Figure 4 (b) presents the evolution of the bubble volume (in 2D: $V_{b}=\pi A B$ ) as function of the volume of the nanowire (i.e., $V_{n}=\pi a b$ ) for each wavelength. From the computed data (vapor bubble for each nanowire and for each wavelength) and by using the same method and the same algorithm, a function $g$ can be obtained through a fit. That function $g$ allows obtaining the relation between the volumes $\ln \left(V_{b}\right)=g\left(\ln \left(V_{n}\right)\right)$. The $g$ function is continuous and strictly increasing; therefore the inverse function $g^{-1}$ also exists. The measurement of the bubble volume $V_{b}$ can be used to determine the volume of the nanowire $V_{n}$ through the relation $\ln \left(V_{n}\right)=g^{-1}\left(\ln \left(V_{b}\right)\right)$. With the two functions $f^{-1}$ and $g^{-1}$, the size and shape of the nanowire can be obtained from the information on the bubble:

$$
\begin{aligned}
\ln \left(V_{n}\right) & =\ln (\pi b a)=\ln \left(\pi b^{2} R_{n}\right) \\
& =\ln \left(\pi b^{2} f^{-1}\left(R_{b}\right)\right)=g^{-1}\left(\ln \left(V_{b}\right)\right) ;
\end{aligned}
$$

consequently,

$$
\begin{aligned}
& b=\left[\frac{\exp \left(g^{-1}\left(\ln \left(V_{b}\right)\right)\right)}{\pi f^{-1}\left(R_{b}\right)}\right]^{1 / 2}, \\
& a=\left[\frac{f^{-1}\left(R_{b}\right) \exp \left(g^{-1}\left(\ln \left(V_{b}\right)\right)\right)}{\pi}\right]^{1 / 2} .
\end{aligned}
$$

Therefore, the measurement of the size and shape of the bubble can be used to obtain information on the geometry of the nanowire and to reconstruct the size and shape of the nanowire.

\section{Conclusion}

The paper focuses on the forming and the evolution of the shape and size of the bubble through a photothermal process between a nanowire of $\mathrm{TiO}_{2}$ immersed in water and an electromagnetic wave. The increase of temperature is related to the geometry of the nanowire which leads to an increase in the shape and size of the bubble. That solution is computed by developing an adaptive remeshing method. That allows to compute with accuracy the temperature by adapting the mesh to the evolution of the bubble. The coupled problem (light, matter, heat) is solved through an adaptive loop process allowing converging to a stable solution and decreasing the number of nodes. The influence of the laser source and the geometrical parameters (wavelength, size, and shape of the nanowire) related to the size and shape of the bubble are presented and analyzed. The aspect ratio and the volume of the bubble can be expressed as function of the aspect ratio and the volume of the nanowire. By solving the inverse problem, two functions are obtained enabling finding the size and shape of the nanowire from the size and shape of bubble. 


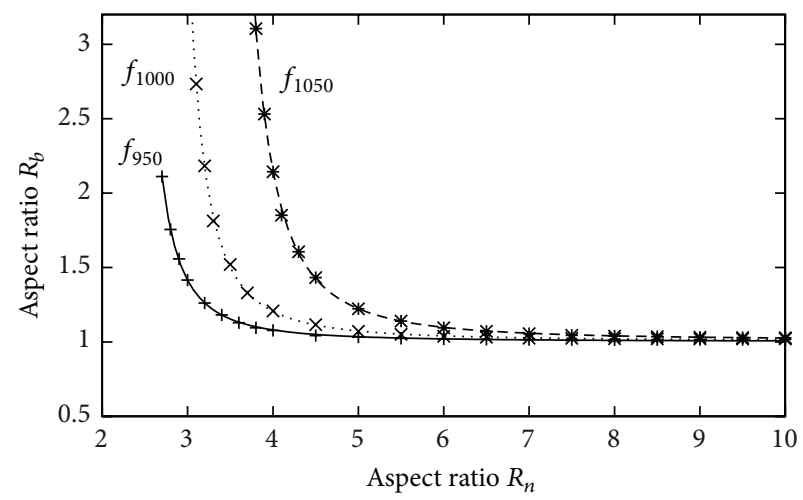

(a)

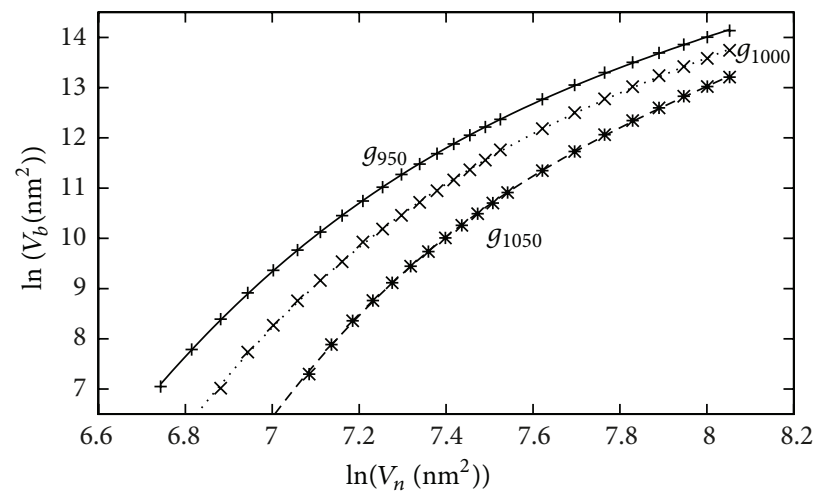

(b)

FIGURE 4: Evolution of (a) the aspect ratio of the bubble $R_{b}$ as function of the aspect ratio of the nanowire $R_{n}$ for the three wavelengths and (b) evolution of the volume of the bubble $V_{b}$ as function of the volume of the nanowire $V_{n}$.

\section{Conflict of Interests}

The authors declare that there is no conflict of interests regarding the publication of this paper.

\section{Acknowledgment}

The authors thank the ANR-2011-NANO-008 NANOMORPH for financial support.

\section{References}

[1] M. Boroski, A. C. Rodrigues, J. C. Garcia, L. C. Sampaio, J. Nozaki, and N. Hioka, "Combined electrocoagulation and $\mathrm{TiO}_{2}$ photoassisted treatment applied to wastewater effluents from pharmaceutical and cosmetic industries," Journal of Hazardous Materials, vol. 162, no. 1, pp. 448-454, 2009.

[2] D. Chatterjee and A. Mahata, "Demineralization of organic pollutants on the dye modified $\mathrm{TiO}_{2}$ semiconductor particulate system using visible light," Applied Catalysis B: Environmental, vol. 33, no. 2, pp. 119-125, 2001.

[3] D. Karamanis, A. N. Ökte, E. Vardoulakis, and T. Vaimakis, "Water vapor adsorption and photocatalytic pollutant degradation with $\mathrm{TiO}_{2}$-sepiolite nanocomposites," Applied Clay Science, vol. 53, no. 2, pp. 181-187, 2011.

[4] G. Bystrzejewska-Piotrowska, J. Golimowski, and P. L. Urban, "Nanoparticles: their potential toxicity, waste and environmental management," Waste Management, vol. 29, no. 9, pp. 25872595, 2009.

[5] B. Nowack, "The behavior and effects of nanoparticles in the environment," Environmental Pollution, vol. 157, no. 4, pp. 10631064, 2009.

[6] D. Lapotko and E. Lukianova, "Laser-induced micro-bubbles in cells," International Journal of Heat and Mass Transfer, vol. 48, no. 1, pp. 227-234, 2005.

[7] D. Lapotko, E. Lukianova, and A. Shnip, "Photothermal responses of individual cells," Journal of Biomedical Optics, vol. 10, no. 1, Article ID 014006, 2005.

[8] D. Barchiesi, T. Grosges, E. Kremer, and M. L. de la Chapelle, "Electromagnetic heat induced in meso-structures: computation of temperature in metallic dimers," PIERS Online, vol. 7, no. 5, pp. 406-410, 2011.
[9] T. Grosges, H. Borouchaki, and D. Barchiesi, "New adaptive mesh development for accurate near-field enhancement computation," Journal of Microscopy, vol. 229, no. 2, pp. 293-301, 2008.

[10] B. Born and E. Wolf, Principle of Optics, Pergamon Press, Oxford, UK, 1993.

[11] T. Grosges, S. Petit, D. Barchiesi, and S. Hudlet, "Numerical modeling of the subwavelength phase-change recording using an apertureless scanning near-field optical microscope," Optics Express, vol. 12, no. 24, pp. 5987-5995, 2004.

[12] T. Grosges, A. Vial, and D. Barchiesi, "Models of near-field spectroscopic studies: comparison between finite-element and finite-difference methods," Optics Express, vol. 13, no. 21, pp. 8483-8497, 2005.

[13] J. Jin, The Finite Element Method in Electromagnetics, John Wiley \& Sons, New York, NY, USA, 1993.

[14] R. Courant, "Variational methods for the solution of problems of equilibrium and vibrations," Bulletin of the American Mathematical Society, vol. 49, no. 1, pp. 1-23, 1943.

[15] P. Silvester and G. Pelosi, Finite Elements for Wave Electromagnetics: Methods and Techniques, IEEE Press, New York, NY, USA, 1994.

[16] R. Fikri, D. Barchiesi, F. H'Dhili, R. Bachelot, A. Vial, and P. Royer, "Modeling recent experiments of apertureless near-field optical microscopy using 2D finite element method," Optics Communications, vol. 221, no. 1-3, pp. 13-22, 2003.

[17] I. Stakgold, Boundary Value Problems of Mathematical Physics, Volumes I and II, Macmillan, New York, NY, USA, 1969.

[18] P. G. Ciarlet, "Basic error estimates for elliptic problems," in Handbook of Numerical Analysis, vol. 2, pp. 17-351, North Holland, Amsterdam, The Netherlands, 1991.

[19] D. Xue and L. Demkowicz, "Modeling of electromagnetic absorption/scattering problems on curvilinear geometries using hp finite/infinite element method," Finite Elements in Analysis and Design, vol. 42, no. 7, pp. 570-579, 2006.

[20] T. Grosges, H. Borouchaki, and D. Barchiesi, "Improved scheme for accurate computation of high electric near-field gradients," Optics Express, vol. 15, no. 3, pp. 1307-1321, 2007.

[21] R. Radovitzky and M. Ortiz, "Error estimation and adaptive meshing in strongly nonlinear dynamic problems," Computer Methods in Applied Mechanics and Engineering, vol. 172, no. 14, pp. 203-240, 1999. 
[22] M. Ainsworth and J. T. Oden, "A posteriori error estimation in finite element analysis," Computer Methods in Applied Mechanics and Engineering, vol. 142, no. 1-2, pp. 1-88, 1997.

[23] P. Laug and H. Borouchaki, "BL2D-V2: mailleur bidimensionnel adaptatif," Report INRIA RT-0275, 2003, http://hal.inria.fr/ inria-00069902.

[24] T. L. Brown and J. A. Rice, "The effect of laser wavelength and power density on the laser desorption mass spectrum of fulvic acid," Organic Geochemistry, vol. 31, no. 7-8, pp. 627-634, 2000.

[25] A. O'Connor, O. Morris, and E. Sokell, "Angular and energy distribution of $\mathrm{Sn}$ ion debris ejected from a laser-produced plasma source, for laser power densities in the range suitable for extreme ultraviolet lithography," Journal of Applied Physics, vol. 109, no. 7, Article ID 073301, 2011.

[26] K. Levenberg, "A method for the solution of certain problems in least squares," Quarterly of Applied Mathematics, vol. 2, pp. 164-168, 1944.

[27] D. Marquardt, "An algorithm for least-squares estimation of nonlinear parameters," SIAM Journal on Applied Mathematics, vol. 11, no. 2, pp. 431-441, 1963.

[28] P. E. Gill and W. Murray, "Algorithms for the solution of the nonlinear least-squares problem," SIAM Journal on Numerical Analysis, vol. 15, no. 5, pp. 977-992, 1978.

[29] V. V. Vasin and G. Y. Perestoronina, “The Levenberg-Marquardt method and its modified versions for solving nonlinear equations with application to the inverse gravimetry problem," Proceedings of the Steklov Institute of Mathematics, vol. 280, no. 1, supplement, pp. 174-182, 2013. 


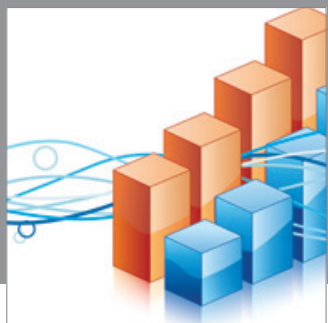

Advances in

Operations Research

mansans

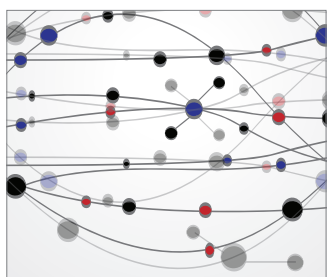

The Scientific World Journal
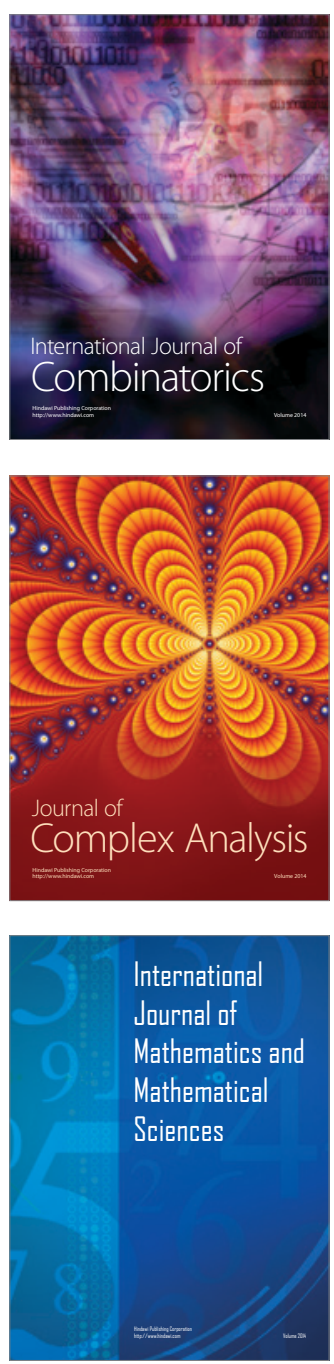
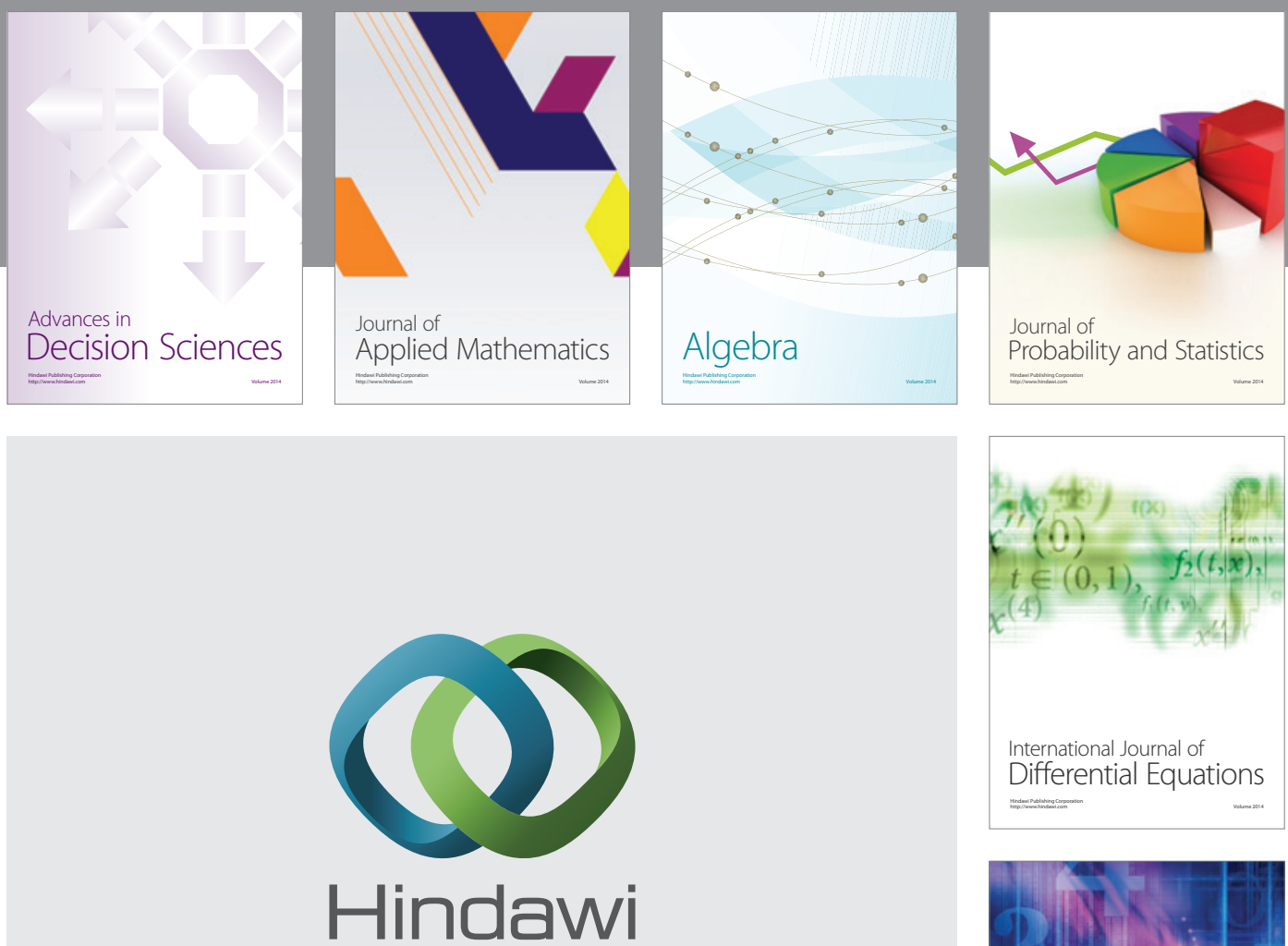

Submit your manuscripts at http://www.hindawi.com
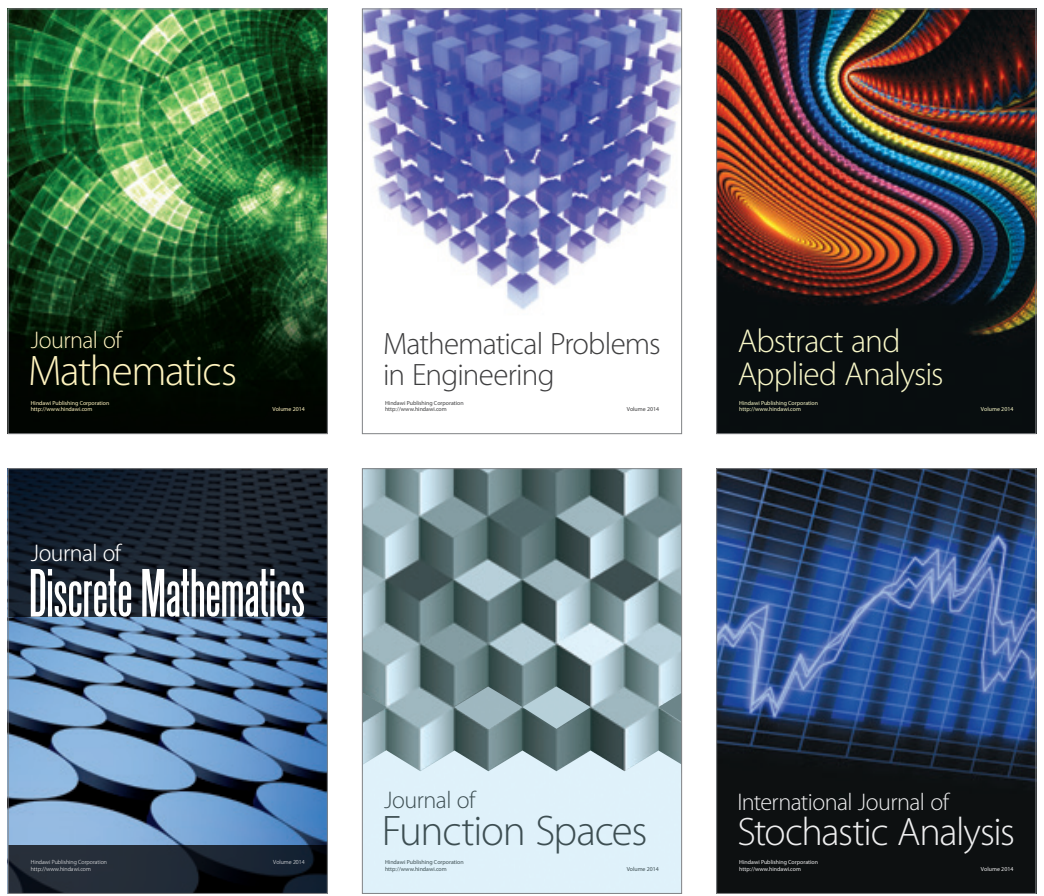

Journal of

Function Spaces

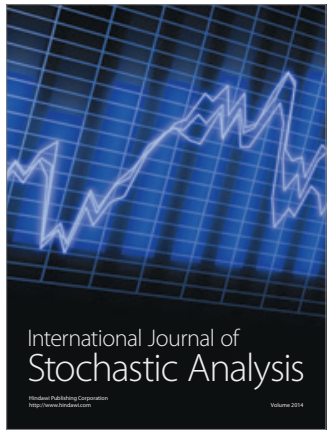

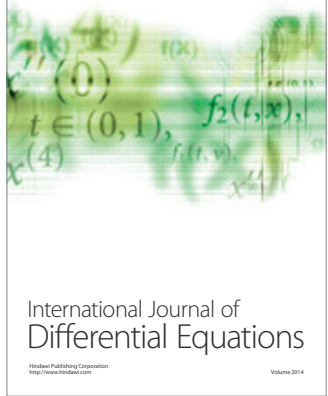
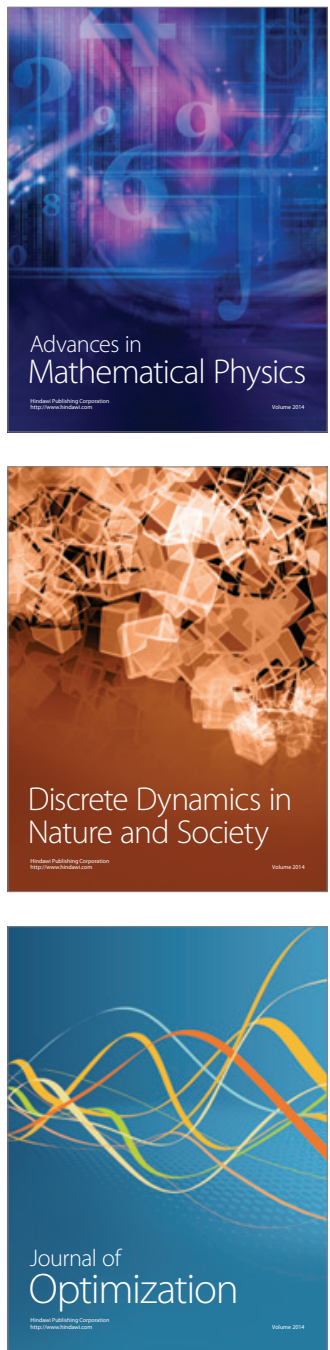